\title{
Kernos
}

Revue internationale et pluridisciplinaire de religion grecque antique

$10 \mid 1997$

Varia

\section{Un instrument divin : la navette, de Platon à Proclus}

\section{Evanghelos A. Moutsopoulos}

\section{OpenEdition \\ Journals}

Édition électronique

URL : http://journals.openedition.org/kernos/663

DOI : $10.4000 /$ kernos. 663

ISSN : 2034-7871

\section{Éditeur}

Centre international d'étude de la religion grecque antique

\section{Édition imprimée}

Date de publication : 1 janvier 1997

Pagination : 241-247

ISSN : 0776-3824

\section{Référence électronique}

Evanghelos A. Moutsopoulos, «Un instrument divin : la navette, de Platon à Proclus », Kernos [En ligne], 10 | 1997, mis en ligne le 12 avril 2011, consulté le 19 avril 2019. URL : http:// journals.openedition.org/kernos/663 ; DOI : 10.4000/kernos.663 


\section{Un instrument divin : la navette, de Platon à Proclus}

Tissage et poterie semblent avoir été parmi les toutes premières techniques qui ont permis la constitution de certains arts et métiers. Le terme même de «métier », appliqué à un appareil complexe, n'est qu'une dénomination par excellence de ce qui, par la suite, devait désigner une acception continue et tant soit peu lucrative (bien qu'originairement limitée à l'intérieur d'une économie fermée) d'un ensemble de procédures exigeant une spécialisation extrême dans le cadre d'une division du travail. Le tissage fut l'effet tout naturel du filage. Clotho, l'une des trois Moires, qui œuvre à allonger ou à écourter le fil de la vie des mortels, est présentée, dans le mythe d'Er ${ }^{1}$, assise sur les genoux d'Ananké, Nécessité : autour du fuseau de celle-ci ${ }^{2}$ sont disposées les planètes dont le mouvement n'est que la condensation des révolutions harmonieuses célestes ${ }^{3}$. La forme de ce fuseau est engendrée et conditionnée par la manière spécifique de l'enroulement du fil sur une baguette. Multiplié, ce fuseau fournira la matière première nécessaire à l'art du tisserand ${ }^{4}$ dont l'ouvrage consiste essentiellement à relier entre eux une série de fils parallèles, les trames, au moyen d'autres fils qui les traversent perpendiculairement, en passant successivement et alternativement au-dessus et au-dessous du plan qu'elles forment.

Ainsi naît le tissu ou « texte ", assemblage de deux groupes de fils parallèles perpendiculairement enchevêtrés les uns par rapport aux autres sur le même plan pour ne former qu'une seule matière solide, plane et continue, se prêtant à maintes manipulations en vue d'usages variés à l'infini. Cet enchevêtrement aurait été pratiquement impossible sans l'utilisation de l'instrument merveilleux entre tous qu'est la navette, descendant très évolué de l'aiguille à coudre, originairement en silex, ayant déjà servi, dans les sociétés paléolithiques, à souder des lésions chirurgicales pratiquées jusqu'à l'intérieur de la boîte crânienne; descendant que Platon, qui en ignore l'origine lointaine, érige en don divin éma-

1 Cf. Platon, Rép., X, 617b-c.

2 Cf. P.-M. SCHUHL, Autour du fuseau d'Ananké, in RA (1930), fasc. 2, p. 58-64.

3 On ne saurait trop rappeler que Mahatma Gandhi, qui pratiquait lui-même le filage, au-delà de toute finalité politique, afin de créer, comme le fait aussi la musique, une atmosphère propice à la méditation, incitait sa pupille Indira Gandhi à l'imiter. Cf. M. GANDHI, Correspondance, V.

4 Le "divin tisserand", encore une figure imaginée par Platon pour désigner le régulateur de la marche du monde; $c f$. PLATON, Pbéd., 87b; Rép., 369d; 374b. 
nant d'Athéna et capable d'enseigner aux humains, par analogie, la technique mentale de la discrimination ${ }^{5}$, capacité qui permet la restructuration de la réalité ${ }^{6}$. Il s'agit, somme toute, d'une technique qui suppose une possibilité de délibération et de décision créative, comparable à la petteia, l'art de prévoir et de préparer ses mouvements lors d'une partie d'échecs, prônée à plusieurs reprises par Platon ${ }^{7}$. Ce qui importe dans ce processus, c'est que les notions de discrimination, de décision et de création sont censées correspondre à des actes précis pouvant être conjugués dans une même pratique.

La volonté de poursuivre et de réaliser un dessein intentionnel déjà établi et toujours en vigueur se solde par le choix délibéré de celles des trames qui, parmi toutes, doivent être, au préalable, séparées des autres : une sur deux (et ce sera le cas le plus fondamental et usuel dans le tissage), deux sur trois, voire trois sur quatre (et l'on retrouve ici certains rapports numériques qui apparaissent déjà dans le domaine de la musique), quand il s'agit de reproduire (en les réalisant) certains dessins en couleurs. Ceci suppose qu'à chaque reprise, des trames différentes sont écartées du plan qu'elles forment avec toutes les autres, pour permettre justement le passage de la navette. Répétition et renouvellement deviennent ainsi les deux mouvements dialectiques complémentaires de cette pratique créative qu'il serait quasiment impossible de réaliser en l'absence de l'instrument, d'importance capitale, qu'elle suppose : la navette. Porteuse d'un fuseau de fil qui se déplace, de forme analogue à celle du fuseau, ce qui laisse supposer que sa fonction fut à l'origine remplie (de manière rudimentaire, certes) par le fuseau lui-même, elle se glisse dans l'espace libre préalablement aménagé entre les trames demeurées immobiles et celles qui, par sélection, ont été provisoirement déplacées en hauteur, pour les réunir par le lien fibral qu'elle leur impose.

Pareille devise pourrait éventuellement être envisagée, sur le plan théorique, sans l'intervention de l'objet navette. Sur le plan pratique, en revanche, elle est, on l'a déjà signalé, rigoureusement exclue par la force des choses. L'objet navette s'impose comme facteur nécessaire et incontournable de la technique de tissage : d'où sa mise en valeur par les textes philosophiques qui en soulignent l'importance; d'où aussi l'accent mis sur le rôle discriminateur et créateur qui lui est assigné, rôle d'instrument médiateur entre la conscience humaine qu'il prolonge dans sa fonction complexe et l'ouvrage matériel à accomplir. C'est ainsi d'ailleurs que le don précieux d'Athéna aux hommes se justifie : chargée, dans

5 Cf. Cratyle, 388c. On ne peut dissocier de ce texte celui du commentaire que lui consacre PROClus, in Crat., 76, 19-30 Pasquali.

6 Cf. Note sur la signification de kairos, in E. MOUTSOPOULOS, Kairos. La mise et l'enjeu, Paris, Vrin, 1991, p. 32-33; ID., Les crises bistoriques, Athènes, Éd. de l'Univ., 1978, p. 5-9.

7 Cf. PLATON, Alcib. I, 110e; Cbarm., 174́b; Gorg, 450d; Pbèdre, 274d; Rép., I, 336b; II, 374c; VI, $487 \mathrm{c}$; Lois, V, 739a; VII, 820c-e; X, 903d. 
un premier temps ${ }^{8}$, de façonner l'espèce humaine en collaboration avec Prométhée ${ }^{9}$, mais aidée d'Hermès, à la doter de moyens de subsistance, Athéna accomplit sa tâche par des dons successifs dont l'invention de la navette n'est pas la moindre.

La notion de navette a une signification importante dans l'œuvre de Platon, surtout en raison de la fonction de cet instrument sur le plan technique du tissage, certes, mais aussi en raison de la signification qu'il acquiert sur le plan de la pensée ${ }^{10}$, comme sur celui de l'action ${ }^{11}$. L'essentiel de la problématique qui s'y rattache est contenu dans le Cratyle. Il s'agit de préparer, à la manière socratique, la qualification du nom comme instrument d'appellation appropriée et discriminante d'un objet. À cet effet, Platon procède en trois étapes distinctes, se servant, au cours de chacune d'elles, de deux exemples analogiques avant de conclure, à chaque reprise, sur la qualité attribuable au nom dont la fonction l'intéresse principalement dans ce dialogue. Le premier exemple est celui de la tarière; le second, celui de la navette ${ }^{12}$. Il est à noter que Platon procède de l'exemple le plus simple à l'exemple le plus complexe, celui qui, justement, remplit davantage les multiples conditions d'analogie lors de la comparaison des notions.

Première étape. Il est question de la nature de l'instrument approprié à être interposé entre le corps agissant et l'ouvrage à accomplir. De coup, c'est sur le critère de la finalité et de l'efficacité de l'instrument que Platon met l'accent ${ }^{13}$ : l'instrument approprié pour l'action de percer, c'est la tarière dont la forme est nécessairement adaptée au travail auquel elle est destinée. Moins simple est le cas de la navette : elle n'est qu'un chaînon - mais combien important et substantiel - dans la suite des conditions nécessaires pour l'accomplissement de l'œuvre du tissage. L'acte de percer suppose plusieurs types d'objets et plusieurs types de tarières ${ }^{14}$; celui de tisser, un métier déjà installé garni d'un dispositif de trames auquel vient s'ajouter et se fondre, au fur et à mesure, un dispositif de chaînes. Si la tarière est susceptible de contribuer à un travail égal dans des conditions diverses, ce qui n'est pas toujours le cas, le travail que la navette contribue à fournir est, de son côté diversifié à l'extrême, ce qui suppose que la

8 Protagoras, $320 \mathrm{~d}$ et sv.

9 Cf. A. THIVEL, Prométhée, personnage romantique, in Diotima, 22 (1994), p. 14-27, notamment les p. 16-17 : à Athènes, Prométhée aurait été le dieu des potiers, c'est-à-dire d'un corps de métier très ancien.

10 Cf. Lysis, 208d; Crat., 388a-c; Polit., 281e, 282c; Lois, VII, $805 \mathrm{e}$.

11 Cf. Crat., 387e, 388a; Soph., 226b, 282b.

12 Cf. Crat., 387e-388a.

13 Il s'agit d'une finalité comportant une fin qui lui est inhérente, car elle vise à une utilité. $C f$. KANT, Critique du jugement, $₫ 17,29$ (Remarque générale) et 62; $f$. E. MouTsopoulos, Forme et subjectivité dans l'esthétique kantienne, Aix-en-Provence, Ophrys, 1964 (Publications des Annales de la Faculté des Lettres et Sciences bumaines d'Aix, 41), p. 121-131.

14 Cf. Crat., $389 \mathrm{c}$. 
navette est destinée à des usages multiples dans le domaine du tissage, et surtout que telle matière à tisser nécessite tel type de navette ${ }^{15}$. Quant au nom ${ }^{16}$ attribué à une chose, son usage est unique, puisqu'il s'applique à cette seule chose à l'exclusion de toute autre, à défaut de quoi il ne saurait remplir convenablement sa fonction et introduirait la confusion dans le langage ${ }^{17}$. La finalité des trois instruments envisagés ici se fait, par conséquent, de plus en plus restreinte : de plus ou moins ample dans le cas de la tarière, elle devient unique dans le cas du nom, le cas de la navette demeurant un cas moyen, une véritable médiété ${ }^{18}$.

Deuxième étape. C'est au tour de la qualité du travail fourni, d'une qualité à l'utilité de l'instrument, d'être envisagée à présent. Le critère de la qualité était, en fait, déjà pris en compte lors de l'examen de la finalité de l'instrument, donc de son caractère approprié à la nature du travail qu'il est censé fournir : une bonne tarière est celle qui perce bien et vite, sans exiger quelque effort démesuré de la part de celui qui la manie; une bonne navette est, elle aussi, facilement maniable : elle se glisse dans les interstices désignés d'avance sans brouiller les fils; un nom est bien choisi quand il sied à la chose dénommée, le maximum d'efficacité étant alors obtenu grâce à l'onomatopée. Or, il s'agit ici d'apprécier la qualité de l'usage de l'instrument par l'usager, qualité qui suppose en l'occurrence l'habileté de l'artisan dans l'utilisation de l'instrument disponible. La qualité, réductible à cette habileté, joue, pour sa part, un rôle déterminant dans la conception et dans la fabrication d'un instrument : une habileté restreinte se traduit par la fabrication d'un instrument rudimentaire qui tout au plus admettra des améliorations successives futures, au fur et à mesure que l'habileté se développera. Il existe indubitablement une dialectique entre le développement de l'habileté et le perfectionnement de l'instrument ${ }^{19}$ : plus l'artisan est avisé, mieux il se servira de son instrument; plus l'instrument est perfectionné, plus l'artisan peut déployer son adresse. On retrouve cette relation à propos de l'utilisation des noms, à la seule différence qu'ici une finesse extrême dans l'usage des termes est requise.

15 Cf. ibid., 389b-c, d, 390b.

16 Cf. Proclus, in Crat., 16, 10-11 P.; cf. E. MOUTSOPOULOS, Les structures de l'imaginaire dans la philosophie de Proclus, Paris, Les Belles Lettres, 1985, p. 76 et la n. 26.

17 Cf. Proclus, in Crat., 5, 20 P.; MOUTSOPOUlos, op. cit. (n. 16), p. 118 et la n. 5.

18 Cf. Crat., 389d-e.

19 Cf. Crat., 390b. On a ainsi relevé que les innovations musicales d'Euripide n'auraient pas été possibles sans le perfectionnement préalable de certains instruments de musique, et qu'à partir d'un certain moment, les compositions de Beethoven n'auraient pu voir le jour, du moins telles quelles, sans l'invention du pianoforte. Cf. Crat., 390b: c'est d'après les instructions du cithariste que le "luthier» fabrique la lyre. On rencontre des métiers horizontaux déjà en Égypte, mais les métiers verticaux furent les plus anciens. Cf. G. GAvaLAS, Filage et tissage au II ${ }^{e}$ millénaire dans les Cyclades, in Actes du III Congrès sur les Cyclades (Santorin, 1995), Athènes, Société d'Études Cycladiques, à paraître. 
Troisième étape. Ici, Platon a recours au critère de la cause efficiente. Outre l'artisan qui utilise l'instrument, son fabricant entre aussi en jeu. Sur l'instruction de l'usager ${ }^{20}$, il façonnera l'instrument: le forgeron, la tarière; le menuisier, la navette. Quant aux noms, instruments du langage, ils sont certes, imposés par l'usage ${ }^{21}$, mais c'est encore un connaisseur, un « législateur » (au sens le plus large du terme, puisqu'il légifère en la matière) qui en désigne l'utilisation $^{22}$. La fabrication de l'instrument est donc possible grâce soit à la copie d'un autre exemplaire de cet instrument, qui sert de modèle à son tour, soit aux prescriptions que son usager futur en donne au fabricant soit, enfin, au modèle intelligible qui s'offre à ce dernier en l'absence de tout autre modèle sensible. De fait, "sur quoi le menuisier a-t-il les yeux quand il fait la navette ? N'est-ce pas sur un objet naturellement propre au tissage ?... Et si la navette se brise pendant la fabrication, en fera-t-il une autre en tenant les yeux sur la navette brisée ou sur telle forme dont il s'inspirait en faisant de la navette qu'il a brisée (...), la navette en soi ${ }^{23}$ ? C'est précisément la fabrication dont l'artisan divin, le démiurge, procède, dans le Timée. Lui aussi construit l'âme du monde les yeux fixés sur le modèle divin ${ }^{24}$. L'identité des situations est frappante et implique des parallèles dans maints domaines. Il en est de même des autres exemples, celui de la tarière et, mutandis mutatis, celui des noms. La suite de la discussion montre que le personnage le mieux placé pour diriger la fabrication des instruments est bien leur usager, celui en tout cas qui, en dialecticien, est à même de procurer au fabricant des instructions pertinentes.

Proclus, de son côté, reviendra à cette problématique ${ }^{25}$ en mettant l'accent sur l'aspect technique de l'usage de la navette, aspect qui relie directement l'instrument avec l'entendement qui le conçoit, mais aussi avec les effets de son usage. L'argumentation vise principalement à circonscrire la fonction du nom ${ }^{26}$, mais Proclus dépasse les considérations platoniciennes pour souligner, à partir de la fonction discriminante du nom ${ }^{27}$, celle de la navette ${ }^{28}$, sans omettre de

20 Cf. ibid.; cf. aussi ibid., 388d-e.

21 Cf. supra et la n. 17.

22 Cf. Crat., 388d-389a.

23 Cf. ibid., 389a-b (trad. L. Méridier); cf. Proclus, in Crat., 65, 15 P. Cf. aussi Platon, Rép., V, $472 \mathrm{e}$.

24 Cf. Timée, 30c-d.

25 Cf. PRoclus, in Crat., 16, 12-27 P.; MOUTSOPoulos, op. cit. (n. 16), p. 77 et la n. $27:$ « Il y aurait lieu d'établir une distinction entre "image du modèle" et "image de la référence au modèle" [...]; les passages parallèles plaident en faveur de la première acception $»$.

26 Cf. in Crat., 16, 5-8 P. : « qui nomme agit; et qui agit le fait moyennant un instrument; par conséquent, qui nomme moyennant un instrument utilise le nom en guise d'instrument; or, des instruments, les uns le sont par nature, les autres parce qu'ils ont été posés comme tels ".

27 Cf. ibid., 18, 5-8 $\mathrm{P}$

28 Cf. ibid., 16, 24-25 P. : «le nom comporte, lui aussi, une puissance adaptée aux signifiés... et, comme il discerne l'essence, il nous procure une connaissance des choses $»$. 
reprendre ce que Platon affirme, à savoir que l'esprit créateur fait preuve d'une activité semblable, puisque d'une part il crée le monde dans son ensemble en gardant le regard fixé sur le modèle intelligible ${ }^{29}$ et que, d'autre part, il attribue des noms en conséquence et conformément à la nature des choses ${ }^{30}$. Pour ce qui est de la puissance à la fois discriminante et créative de la navette (kerkis), Proclus recourt à l'image homérique de Circé (Kirkè; il y a ici plus qu'un jeu de mots) en train de tisser l'ensemble de la vie à l'intérieur du châssis (tetrastoikbon) de son métier, tout en rendant le monde sublunaire harmonieux grâce à ses chants ${ }^{31}$. Cette double fonction, qui constitue l'essence même du tissage, Athéna Erganè l'a révélée aux humains ${ }^{32}$ par l'entremise de la nature au sein de laquelle tout se distingue et se compose dans l'harmonie de ce qui est né et de ce qui demeure éternel, du mortel et de l'immortel, du corporel et de l'incorporel, du sensible et de l'intelligible ${ }^{33}$. C'est d'ailleurs de la même manière que la navette «sépare les genres qui forment les êtres, pour qu'en même temps que leur composition leur division se perpétue afin que leur existence authentique soit préservée ${ }^{34}$. Le miracle de la composition harmonieuse consiste justement dans le maintien des différences à l'intérieur de leur fusion ${ }^{35}$.

Fabricant et usager se rencontrent au niveau du modèle intelligible qu'ils réalisent sur le plan sensible en raison de la dialectique qui émane de leur collaboration ${ }^{36}$. Et puis, qu'est-ce que l'art sinon « l'artificiel hors de la matière, dans l'âme de l'artisan ${ }^{37}$ ? La raison de la navette, par exemple telle que le tisserand la conçoit, c'est sa forme spécifique, mais aussi la finalité pratique de son usage $^{38}$. La navette devient, par sa forme, sa finalité et son utilité, l'image symbolique du pouvoir discrétionnaire des dieux qui procèdent à la distinction du tout, avant de tout recomposer. Ce pouvoir s'imprime sur la texture du tissu, qui en devient l'image sensible ${ }^{39}$ : les chaînes qui s'unissent aux trames reproduisent la création des espèces dans l'univers ${ }^{40}$. Proclus avance l'opinion (moi dokousi) que, sur le plan de la pensée philosophique, cette symbolique se concrétise en

29 Cf. PLATON, Crat., 389a et suiv.; Timée, 36c.

30 Cf. Proclus, in Crat., 20, 22-24 P. Proclus n'hésite pas à recourir au témoignage de sources tardives qu'il juge plus affirmatives; $c f$. ibid., 20, 26; 21, 2 P; cf. Oracles chaldaïques, fr. 43 (p. 78 E. DES PlaCES); 50 (49, p. 79 E. DES PlaCeS); cf. PROClus, in Tim., III, 14, 13-14 Diehl.

31

32

33

34

35

36

37

38

39

40

Cf. in Crat., 22, 28-30 P.

Cf. ibid, 22, 20 et sq. P.; cf. supra et la n. 9.

Cf. in Crat., 22, 20-23 P.

Ibid., 22, 25-28 P.

Cf. ibid., 24, 9-13 P.

Cf. ibid., 22, 28; 23, $25 \mathrm{P}$.

Cf. in Crat., 22, 28-30 P.

Cf. ibid., 22, 20 et sq. P.

Cf. ibid., 22, 20 et sq. P.

Cf. supra et la n. 38. 
tant qu'analogie ${ }^{41}$. C'est ainsi que Platon (comme Proclus, dans son Commentaire) se référait à la navette dans le Cratyle pour désigner la puissance créative et ses processus ${ }^{42}$, et aux chevaux dans le Phèdre ${ }^{43}$ pour désigner telle ou telle puissance de l'âme ${ }^{44}$.

On tentera de conclure en affirmant que dans la tradition philosophique qui remonte à Platon et, au-delà de celui-ci, à une tradition culturelle plus ou moins diffuse, l'instrument essentiel du tissage, la navette, représente le processus complexe de la création, mieux : la combinaison de deux processus créationnels alternatifs, celui de la discrimination et celui de la composition; mais que Proclus se serait plus clairement prononcé sur cette question, en mettant en évidence la dialectique de la composition des mixtes ${ }^{45}$, telle que Platon l'avait envisagée dans ses dialogues tardifs, sur le plan de la création esthétique, au sens à la fois de tecbnè et de tecbniké : analogie, certes, ainsi que Proclus le suggère; mais analogie qui comporte, indubitablement, des prolongements symboliques. Dans cette perspective, il ne semble pas exagéré de prétendre que l'instrument navette, don divin, est érigé en devise concrète de l'ingéniosité productive, mais aussi de la conception ontologique de la créativité divine et humaine.

E. MOUTSOPOULOS

Hypsilantou, 40

GR - 11521 ATHÈNES

41 Cf. in Crat., 22, 28-30 P. : « l'analogie n'est pas un rapport entre idée et image ».

42 Cf. E. MOUTSOPOULOS, Alternative Processes in Artistic Creation, in Proceedings of the 8th International Wittgenstein-Symposium (Kircbberg am Wechsel, 1982), Part I, Wien, Hölder, 1984, p. 367-377.

43 Cf. PLATON, Pbèdre, $246 \mathrm{a}$ et sq.

44 Cf. PROClus, in Crat., 24, 28-31 P.

45 Cf. N.-I. BOUSSOUlAs, L'être et la composition des mixtes dans le Pbilèbe de Platon, Paris, P.U.F., 1952; Étude sur l'estbétique de la composition platonicienne des mixtes, in RMM, 64 (1960), p. 442-448 et 65 (1961), p. 142-158; L'esthétique platonicienne, in Actes du IVe Congrès International d'Estbétique, Athènes, 1960, p. 748-754. Cf. E. MOUTSOPOUlOS, La musique dans l'ceuure de Platon, Paris, P.U.F., $1989^{2}$, p. 358-363. 